\title{
Uso da ferramenta PreText para mineração de textos extraídos do NCBI para estudo epistemológico da Informática em Saúde
}

\author{
Eliane Colepícolo ${ }^{1}$ \\ Edson Takashi Matsubara ${ }^{2}$ \\ Alex Esteves Jaccoud Falcão ${ }^{1}$ \\ Ivan Torres Pisa ${ }^{1}$
}

\begin{abstract}
Resumo: Este artigo apresenta a utilização da ferramenta PreText como técnica auxiliar a uma pesquisa sobre epistemologia da Informática em Saúde (IS), que visa inferir se a IS se caracteriza como ciência, tecnologia, tecnociência ou arte. O PreText tem por objetivo realizar pré-processamento de textos, transformando-os em um formato estruturado, usando a abordagem bag-of-words, e foi aplicado aos metadados de 437.289 resumos de artigos científicos extraídos da base PubMed Central. Os resultados do processamento foram exportados para uma base de dados e relacionados a um coleção de termos de um tesauro especializado em IS construído pelos autores, denominado EpistemIS, e aos metadados dos artigos para geração de estatísticas. Tais relações possibilitaram compreender a epistemologia da IS, inferindo que esta é uma tecnociência interdisciplinar que atua nos domínios das Ciências da Vida, Ciências da Saúde e Cuidado em Saúde.
\end{abstract}

\begin{abstract}
This article presents the PreText tool as auxiliary technique to a research on Health Informatics (HI) epistemology, which aim to understand, through text mining, among other methods and techniques, if the HI is characterized as science, technology, tecnoscience, or art. The PreText is used to execute texts preprocessing, transforming these texts in structured format using a bag-of-words approach. It was applied in 437,289 abstracts of articles extracted from PubMed Central database. The results were exported to a database and related to a terms collection from a specialized thesaurus in Medical Informatics built by the authors, dubbed EpistemIS, and to the articles metadata for statistics generation. These relations make possible a HI's epistemological study, concluding that this is an interdisciplinary technoscience in Biological Sciences, Health Sciences, and Health Care domains.
\end{abstract}


Uso da ferramenta PreText para mineração de textos extraídos do NCBI para estudo epistemológico da Informática em Saúde

\section{Introdução}

A Inteligência Artificial (IA), como subárea da Ciência da Computação, vem sendo largamente utilizada em uma série de aplicações científicas, tecnológicas, industriais e até mesmo no comércio e serviços. A IA pode ser definida como a ciência e engenharia de construção de máquinas inteligentes, e em particular, de programas de computadores inteligentes [1]. Podemos dizer que normalmente um computador é programado para emular um tipo de inteligência, e não muitos, pois um programa de IA é desenvolvido ad hoc, ou seja, para resolver problemas específicos de um domínio também específico [2]. Para a IA, não existem problemas, nem soluções típicas, mas existem técnicas que permitem definir o tipo de sistema que se deseja desenvolver. Um dessas técnicas é o Processamento de Linguagem Natural (PLN), cujo principal objetivo é transformar a linguagem natural em linguagem artificial.

A linguagem é definida [3] como a faculdade natural de usar uma língua, ao passo que a língua constitui algo adquirido e convencional, um sistema de signos distintos correspondentes a idéias distintas. A linguagem artificial surge como uma forma de transformar a linguagem natural legível por computador, o qual está vinculado à Lingüística Computacional. O PLN tem por objetivo desenvolver uma teoria computacional da linguagem, usando notações de algoritmos, estruturas de dados e técnicas da Ciência da Computação [4].

Neste artigo utilizamos a mineração de textos, que utiliza recursos de PLN e lida com a extração de conhecimento e informação em bases textuais [5]. Há vários tipos de mineração de textos, entre eles: sumarização, para resumo de artigos; clustering, para agrupamentos de documentos; classificação de ou associação entre dados, informação, conhecimento e documentos. Para que ocorra a mineração de textos, há uma etapa de recuperação de informação, que envolve a coleta dos textos a serem minerados, cujo conjunto é denominado corpus. A busca estratégica para construção deste corpus pode envolver o uso de operadores lógicos e de proximidade, mas torna-se mais eficaz se baseada em tesauros [6], que podem ser definidos como vocabulários controlados que representam hierarquias, relações de equivalência, pertinência e associações entre os termos. Existem muitos softwares para mineração de textos disponíveis no mercado, entre eles, o PreText ${ }^{\odot}$, ferramenta desenvolvida pelo pesquisador Edson T. Matsubara, no Laboratório de Inteligência Computacional (LABIC), do Instituto de Ciências Matemáticas e Computação da Universidade de São Paulo [7]. O PreText vem sendo utilizado em outros trabalhos científicos como ferramenta para mineração de textos, tais como os desenvolvidos por Martins [8] e por Matsubara [9].

Neste trabalho, a mineração de textos com PreText foi utilizada como técnica auxiliar a uma pesquisa que resultou em uma dissertação de mestrado [10] sobre a epistemologia da Informática em Saúde (IS). A epistemologia é o ramo da filosofia da ciência que trata da cientificidade de uma área do conhecimento, é a ciência da ciência [11]. Assim, a pesquisa 
teve como objetivo compreender se IS se comporta como ciência, tecnologia, tecnociência ou arte, utilizando um conjunto de métodos integrados. O objetivo da mineração com PreText na pesquisa foi identificar em um corpus de artigos científicos associados a um tesauro especializado em IS os termos pertinentes à área de IS, cujos resultados possibilitaram inferências sobre a epistemologia da IS. Este artigo apresenta descrição sobre o uso do PreText e os resultados obtidos na pesquisa.

\section{Materiais e Métodos}

Os materiais (Figura 1) envolvidos na mineração de textos da pesquisa sobre a epistemologia da IS foram os seguintes: a) corpus de análise de metadados de artigos científicos da área no idioma inglês; b) ngramas ${ }^{1}$ de resumos destes artigos, após a mineração de textos; c) um tesauro especializado em IS, denominado EpistemIS, construído por Colepícolo, Falcão e Pisa [10]. Os métodos utilizados foram: 1) a mineração de resumos dos artigos do corpus, usando PreText, que resultou no conjunto de ngramas dos termos dos resumos; 2) a aplicação do algoritmo de Porter [12] ao conjunto de termos do tesauro, que resultou no conjunto de ngramas dos termos do tesauro; 3) a elaboração de estatísticas envolvendo os ngramas dos resumos, os ngramas do tesauro e os metadados dos artigos do corpus, as quais permitiram inferências sobre a epistemologia da IS.

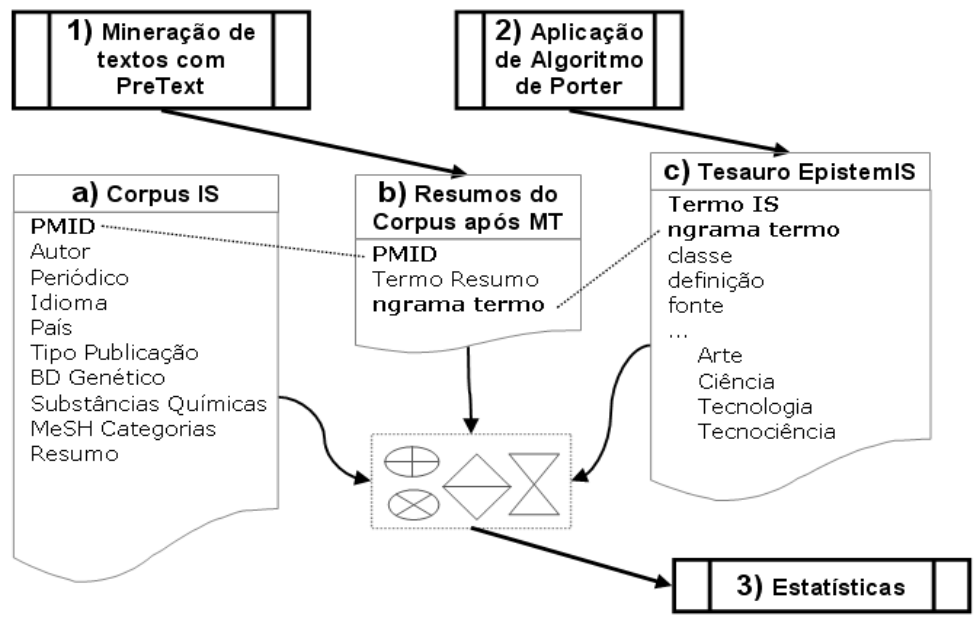

Figura 1 - Materiais e métodos.

${ }^{1}$ nGramas são seqüências de $\mathrm{n}$ termos ou radicais de termos (stems) consecutivos utilizados em mineração de texto para encontrar termos coincidentes em conjuntos de dados diferentes. 
Uso da ferramenta PreText para mineração de textos extraídos do NCBI para estudo epistemológico da Informática em Saúde

\section{Corpus de Análise}

Para o corpus de análise foi selecionado um conjunto de 437.289 artigos científicos, provenientes da base de dados PubMed Central [13], disponível no National Center Biotechnology Information (NCBI), um portal da National Library of Medicine (NLM), do período de 1997 e 2006. A pesquisa bibliográfica que resultou no corpus foi realizada a partir de uma estratégia de busca com 2.538 termos de Medical Subject Headings (MeSH) [14] (Quadro 1), provenientes das categorias consideradas próximas ao tema IS, utilizando o operador booleano "OR", com objetivo de recuperar todo texto indexado por qualquer um dos termos selecionados.

Quadro 1 - Categorias MeSH utilizadas na pesquisa.

\begin{tabular}{|l|l|}
\hline Categoria MeSH & \multicolumn{1}{|c|}{ Área } \\
\hline E05 & Investigative Techniques \\
\hline E07 & Equipment and Supplies \\
\hline L01 & Information Science \\
\hline N01 & Population Characteristics \\
\hline N02 & Health Care Facilities, Manpower, and Services \\
\hline N03 & Health Care Economics and Organizations \\
\hline N04 & Health Services Administration \\
\hline N05 & Health Care Quality, Access, and Evaluation \\
\hline
\end{tabular}

A coleta do corpus envolveu a extração de metadados dos artigos em XML e conversão destes para uma base de dados relacional, utilizando algoritmos e códigos de programação em linguagem PHP. A extração dos metadados dos artigos foi feita por meio de um utilitário, chamado efetch, disponível no site do NCBI, próprio para obtenção de grandes quantidades de registros de suas bases de dados [15]. O utilitário (Quadro 2) consiste em uma URL base a qual podem ser aplicados parâmetros específicos para recuperação de registros das bases NCBI, a partir de seus respectivos PMIDs, ou seja, os códigos identificados únicos de cada artigo no sistema. A seguir, os metadados foram exportados para uma base de dados relacional.

Quadro 2 - Utilitário efetch do NCBI.

\begin{tabular}{|c|l|l|}
\hline Utilitário & efetch \\
\hline \multirow{2}{*}{ Descrição } & $\begin{array}{l}\text { URL parametrizável que recupera registros das bases específicas nos formatos de } \\
\text { apresentação e de saida especificados pelos parâmetros }\end{array}$ \\
\hline \multirow{2}{*}{ URL base } & $\begin{array}{l}\text { http://eutils.ncbi.nlm.nih.gov/entrez/eutils/efetch.fcgi?db= } \\
\text { pubmed\&id=PMID\&retmode=xml\&report=medline }\end{array}$ \\
\hline \multirow{5}{*}{ Parâmetros } & \# (id=NNNNNN) or (query_key=NNN) & where NNN - number in the history \\
\cline { 2 - 3 } & $\begin{array}{l}\text { \# db=db_name } \\
\text { \# report=[docsum, brief, abstract, citation, medline, } \\
\text { asn.1, mlasn1, uilist, sgml, gen] }\end{array}$ & (Optional; default is asn.1) \\
\cline { 2 - 3 } & \# mode=[html, file, text, asn.1, xml] & (Optional; default is html) \\
\cline { 2 - 3 } & \# dispstart=first element to display, from 0 to -1 & (Optional; default is 0) \\
\cline { 2 - 3 } & \# dispmax - number of items to display & (Optional; default is all elements) \\
\hline
\end{tabular}

Um dos metadados dos artigos do corpus é o resumo, ao qual foi aplicada a mineração de textos usando a ferramenta PreText, que resultou nos ngramas de todos os termos compostos por 1, 2 e 3 palavras dos resumos do corpus. 


\section{Tesauro EpistemIS}

Foi desenvolvido um tesauro especializado em IS, denominado EpistemIS, com base no cabeçalho MeSH [14] transformado em tesauro MeSH [16] e integrado a conceitos e definições da literatura em IS. O tesauro EpistemIS contém uma série de atributos, entre os quais a classificação de cada um dos termos do tesauro em ciência, tecnologia, tecnociência ou arte (Figura 3). Por ser baseado no MeSH, tesauro validado por especialistas da NLM, e baseado em conceitos e definições provenientes da literatura especializada em IS, entende-se que estes critérios por si só, já asseguram a validação do tesauro EpistemIS.

As principais obras utilizadas para a extração de conceitos e definições da Informática em Saúde foram o "Handbook of Medical Informatics" de Van Bemmel, publicada em 1999 [17] e o "Biomedical Informatics: computer Applications in Health Care and Biomedicine" de Shortliffe \& Cimino, publicada em 2006 [18].

Para a classificação dos termos do EpistemIS, tomamos como base definições de ciência, tecnologia, tecnociência ou arte, denominados Metaconceitos da Ação e do Pensamento Humanos (MAPHs), baseadas em estudo epistemológico apresentado na dissertação de Colepícolo [10] (Figura 2):

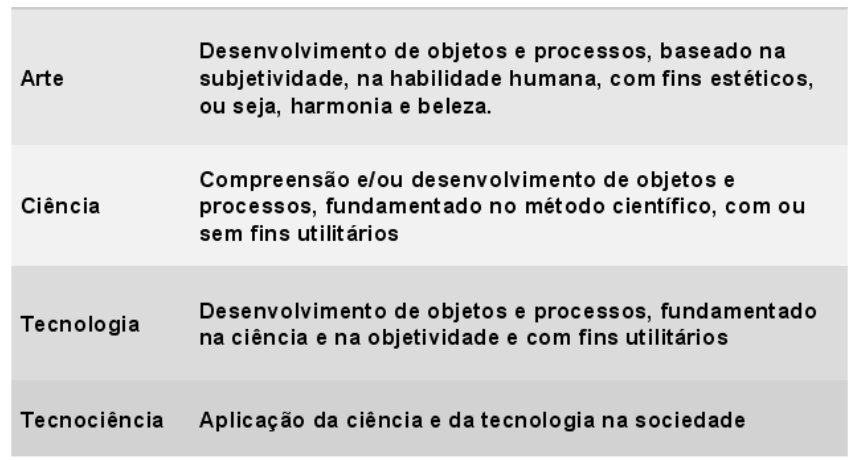

Figura 2 - Definições dos MAPHs.

Também foram feiras classificações dos conceitos de cada termo do tesauro EpistemIS quanto à base conceitual, tipo conceitual, função e objetivo conceituais e quanto ao tipo de conceitos teóricos, com base no referencial teórico de Mário Bunge [19, 20]. 
Uso da ferramenta PreText para mineração de textos extraídos do NCBI para estudo epistemológico da Informática em Saúde

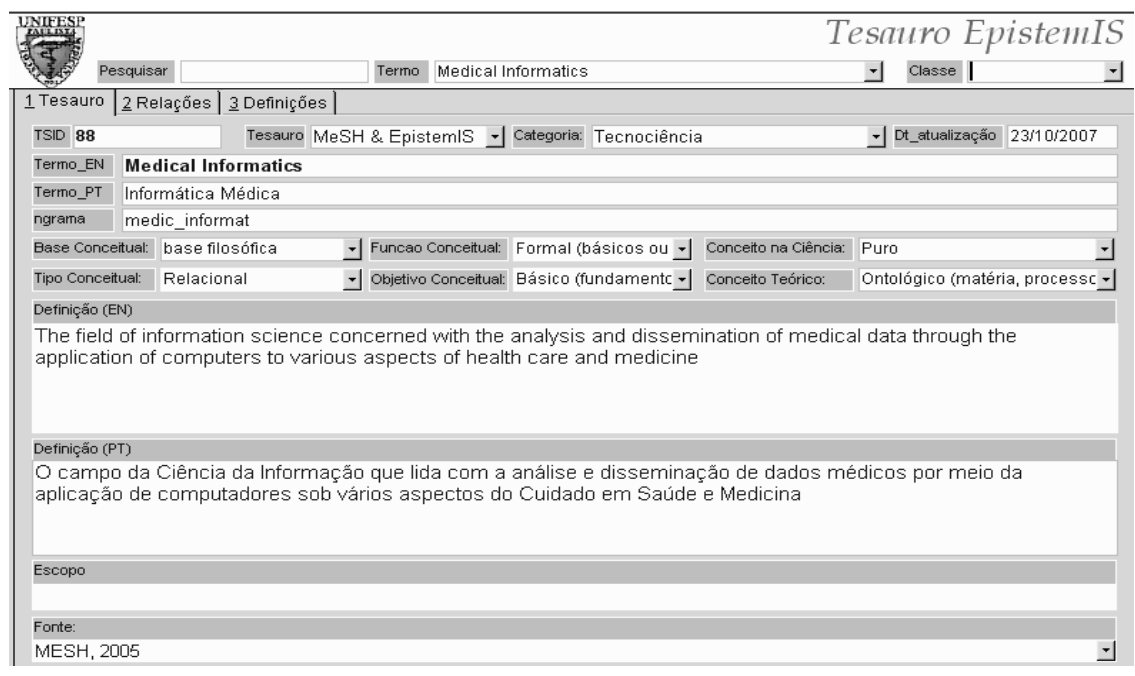

Figura 3 - Tela do programa de consulta ao tesauro EpistemIS.

A associação entre o corpus e o tesauro EpistemIS foi feita por meio de ngramas que possibilitaram identificar termos coincidentes entre os ngramas dos resumos e os ngramas dos termos do tesauro. Com o corpus e o tesauro EpistemIS disponíveis, foi possível iniciar o processo de mineração de textos usando o software PreTexT.

\section{Mineração de Textos com PreText}

O PreTexT é uma ferramenta computacional para mineração de textos desenvolvida na linguagem de programação Perl, que tem por objetivo realizar pré-processamento de textos, transformando estes textos em um formato estruturado legível por algoritmos de Aprendizado de Máquina, entre outras aplicações. Este pré-processamento baseia-se na abordagem bag-of-words, que transforma dados em um formato não estruturado para um formato estruturado, usando o recurso de "tabela atributo-valor", na qual cada documento é representado como um vetor das palavras que ocorrem no documento ou como frases ou sentenças [7, 9].

No caso do PreText, foi proposta uma nova métrica para avaliar a relevância de um termo dentro de um documento, semelhante à $t f i d f$, denominada term frequency linear (tflinear) [7, 9], baseada na métrica term frequency - inverse document frequency. Na tflinear, o fator de ponderação é linear e não logarítmico, variando entre 0 e 1, ou seja:

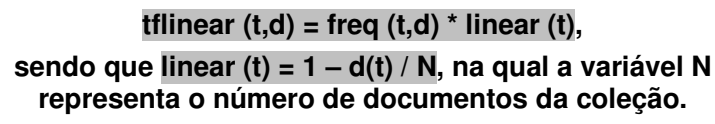


Além disso, foi proposta uma nova medida denominado smooth, que é ativada quando o fator de ponderação é igual a zero, ou seja, quando um termo aparece em todos os documentos. Quando ativado, ele aumenta temporariamente em 10\%; desse modo, o fator não pode ser igual a zero. Maiores detalhes sobre as técnicas de mineração utilizadas no PreText podem ser encontradas na dissertação de mestrado de Matsubara [9].

Um dos problemas encontrados na mineração de textos é a dimensionalidade dos atributos de um corpus, ou seja, a relação entre o número de documentos da coleção, a quantidade de termos que aparece no total da coleção e a quantidade de termos que aparece em cada documento, que pode resultar numa matriz esparsa. Para resolver este problema, o PreText utiliza 3 técnicas de redução da dimensionalidade dos atributos, que são: algoritmos de Stemming, Lei de Zipf e os cortes de Luhn [7]. A Figura 4 mostra um exemplo de parâmetros e resultados obtidos com a execução do PreText. Os arquivos resultantes da mineração com PreText foram convertidos para formato de banco de dados relacional.

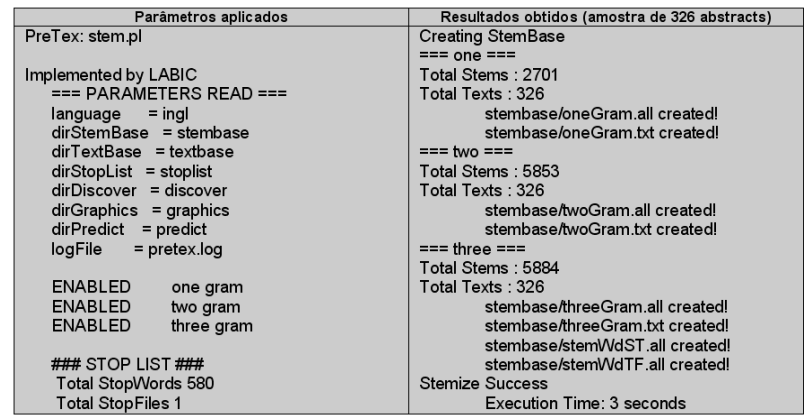

Figura 4 - Parâmetros e resultados da execução do PreText.

\section{Aplicação do Algoritmo de Porter ao Tesauro EpistemIS}

Para tornar possível a associação entre os termos do tesauro EpistemIS e os termos obtidos dos resumos dos artigos do corpus foi aplicado o algoritmo de Porter [12] sobre os termos do tesauro EpistemIS, transformando-os em ngramas no padrão PreText (Figura 5). Este algoritmo costuma ser utilizado para termos em inglês, como é o caso dos termos do tesauro e do corpus utilizados neste trabalho.

\begin{tabular}{|l|l|l|} 
12.11.08.05 & HCl in Health Care & hci_health_care \\
\hline 12.11.08.05.01 & Health Care Information Framework & health_care_inform \\
\hline 12.11.09 & Health Informatics & health_inform \\
\hline 12.11.09.01 & Health Informatics Computing & health_inform_comput \\
\hline 12.11.09.01.01 & Health Informatics Concepts & health_inform_concept \\
\hline 12.11.09.02 & Health Informatics Applications & medic_informat_applic \\
\hline 12.11.09.02.01 & Telematics & telematic \\
\hline 12.11.09.02.02 & Biomedical Engineering & biomed_engin \\
\hline 12.11.09.03 & Bioinformatics & bioinformat \\
\hline 12.11.09.03.01 & Pathway Informatics & pathwai_inform \\
\hline 12.11.09.03.02 & Microarray Technology & microarrai_technolog \\
\hline
\end{tabular}

Figura 5 - Exemplos de ngramas de conceitos do tesauro EpistemIS. 
Uso da ferramenta PreText para mineração de textos extraídos do NCBI para estudo epistemológico da Informática em Saúde

Com a mineração dos resumos e importação dos seus respectivos ngramas e a criação dos ngramas do tesauro EpistemIS foi possível fazer a associação entre ngramas de resumos e de termos EpistemIS para geração de estatísticas sobre a epistemologia da IS (Figura 6), utilizando-se ferramenta de banco de dados relacional, relacionando-se os ngramas do tesauro aos ngramas do corpus.

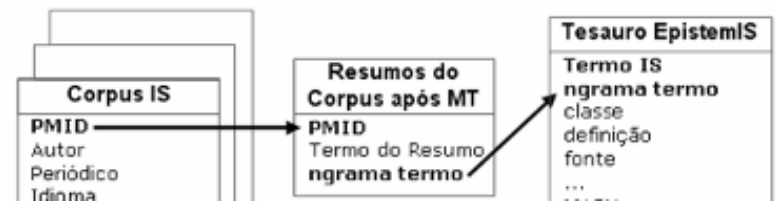

Figura 6 - Relacionamento entre objetos da base de dados para Estatísticas

\section{Resultados}

O uso dos métodos de mineração de resumos do corpus e aplicação do algoritmo de Porter ao tesauro EpistemIS resultou em um grande conjunto de dados armazenados em banco de dados relacional que foram relacionados, agrupados e classificados, resultando em uma série de estatísticas. Apenas resultados mais relevantes para analisar a epistemologia da Informática em Saúde são apresentados neste artigo, especialmente quanto à classificação dos conceitos do tesauro em arte, ciência, tecnologia ou tecnociência.

Os gráficos de evolução da base conceitual (Figura 7) e do objetivo conceitual (Figura 8) dos termos do tesauro EpistemIS em relação aos aspectos arte, ciência, tecnologia e tecnociência mostram que a IS utiliza a pesquisa científica e o desenvolvimento tecnológico interdisciplinares para gerar soluções de aplicação próprias para fins práticos. A partir destas soluções, a área cria seu próprio corpo de conhecimento, mas com objetivos utilitários.

A base específica ou interdisciplinar da IS é científica (Figura 7, gráfico 1), enquanto seu corpo de conhecimento é tecnocientífico (Figura 7, gráfico 2). Os objetos e fatos de domínio da área são tecnológicos e científicos (Figura 7, gráficos 1 e 3) e a sua problemática é tecnológica (Figura 7, gráfico 3). Os conceitos científicos da IS têm objetivo metalógico, básico e interpretativo (Figura 8, gráfico 1), mostrando o caráter interdisciplinar e normalizado da área, enquanto os conceitos tecnológicos (Figura 8, gráfico 3) e artísticos (Figura 8, gráfico 4) têm objetivo básico e descritivo. Nota-se que há preocupação da comunidade científica em IS em definir suas tecnologias e os passos envolvidos no desenvolvimento de sistemas e das interfaces dos sistemas desenvolvidos. Os conceitos tecnocientíficos (Figura 8, gráfico 3) têm objetivo básico, mostrando a capacidade da IS em solucionar problemas das Ciências da Saúde e Cuidado em Saúde na sociedade. 
Uso da ferramenta PreText para mineração de textos extraídos do NCBI para estudo epistemológico da Informática em Saúde
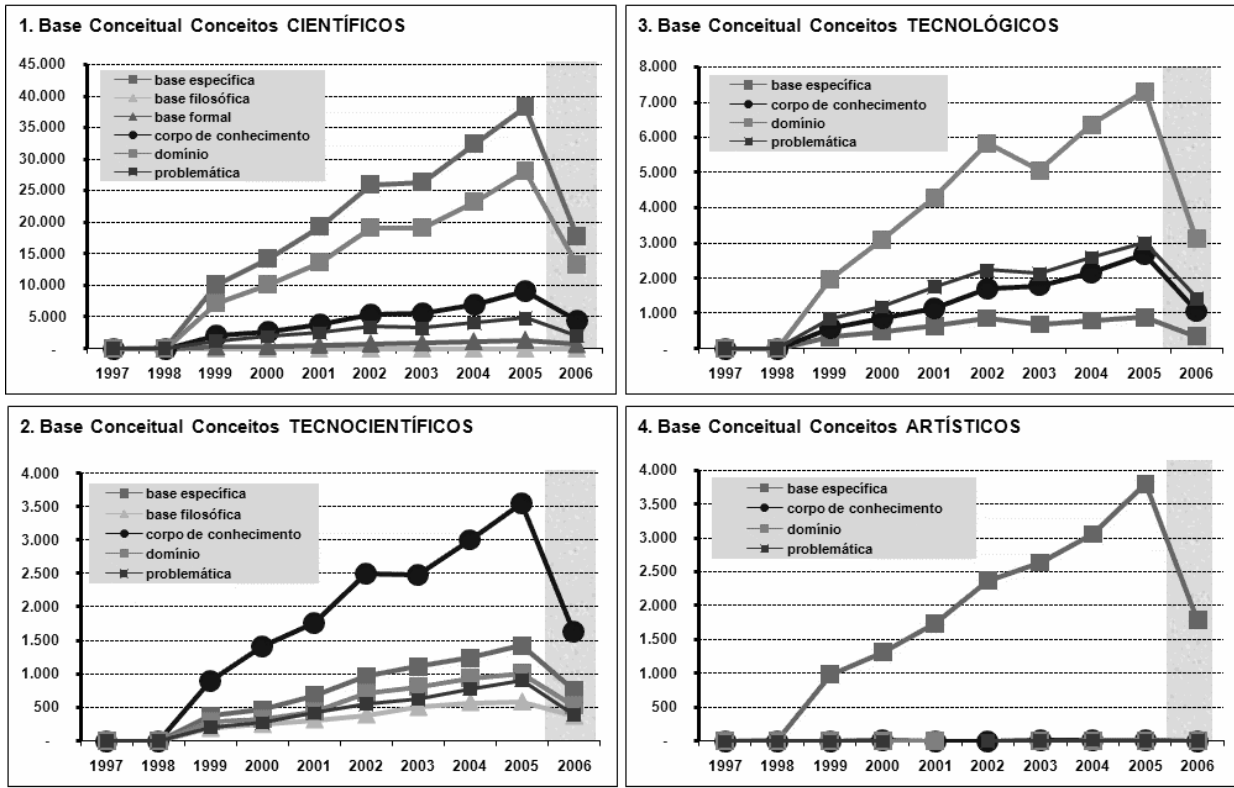

Figura 7 - Medições da Base conceitual da IS por tipo de conceito: 1) científicos;

2) tecnocientíficos; 3) tecnológicos; 4) artísticos.
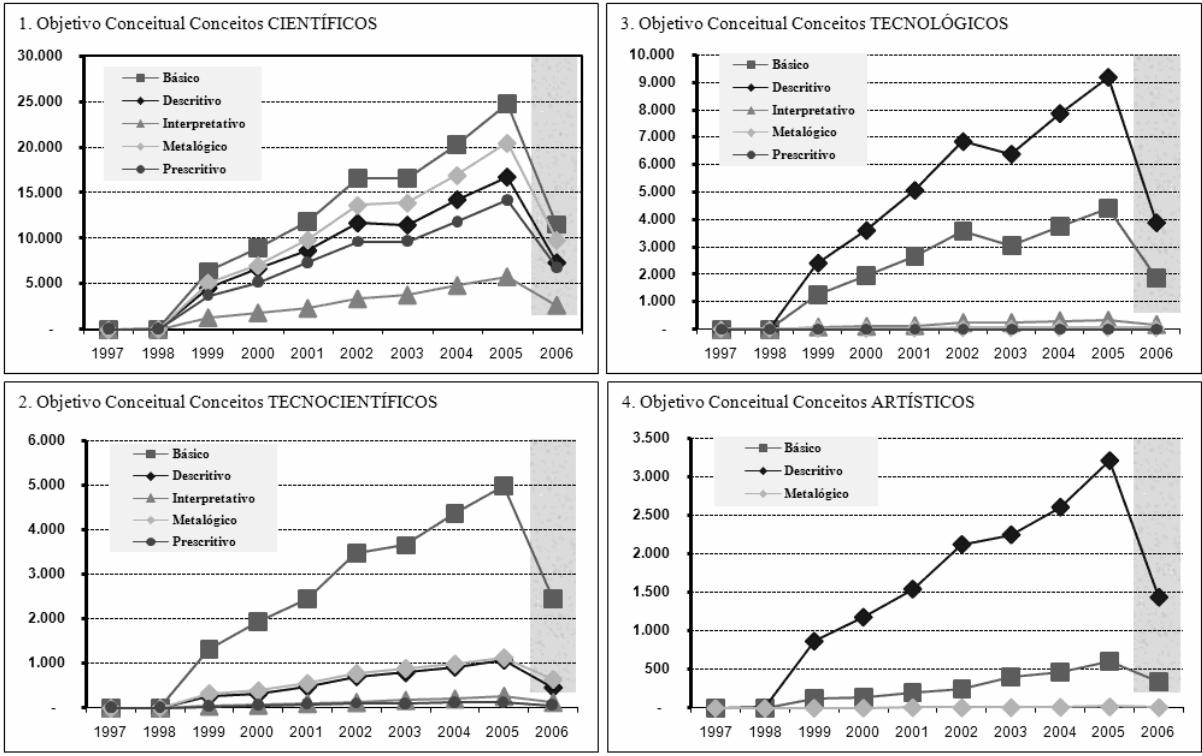

Figura 8 - Medições do objetivo conceitual da IS por tipo de conceito: 1) científicos; 2) tecnocientíficos; 3) tecnológicos; 4) artísticos. 
Uso da ferramenta PreText para mineração de textos extraídos do NCBI para estudo epistemológico da Informática em Saúde

Do cruzamento entre metadados de artigos do corpus, ngramas dos resumos e ngramas do tesauro EpistemIS obtivemos gráficos de artigos publicados por autor individual, autor coletivo, periódico, idioma, banco de dados genético e substâncias químicas (Figura 9) associados aos aspectos ciência, tecnologia, tecnociência e arte. Por exemplo, o total de autores individuais que publicam artigos com ênfase científica é de 2.292.569. Os artigos associados a bancos de dados genéticos são na maioria de caráter científico e tecnológico, o que reforça a tendência da IS em se aliar à Biologia e Ciências Biomédicas para transformarse em Informática Biomédica. Notamos nestes gráficos um padrão no qual prevalece o aspecto científico, seguido pelo tecnológico e tecnocientífico, sendo o artístico quase insignificante, o que mostra que na prática a IS publica mais ciência e tecnologia.

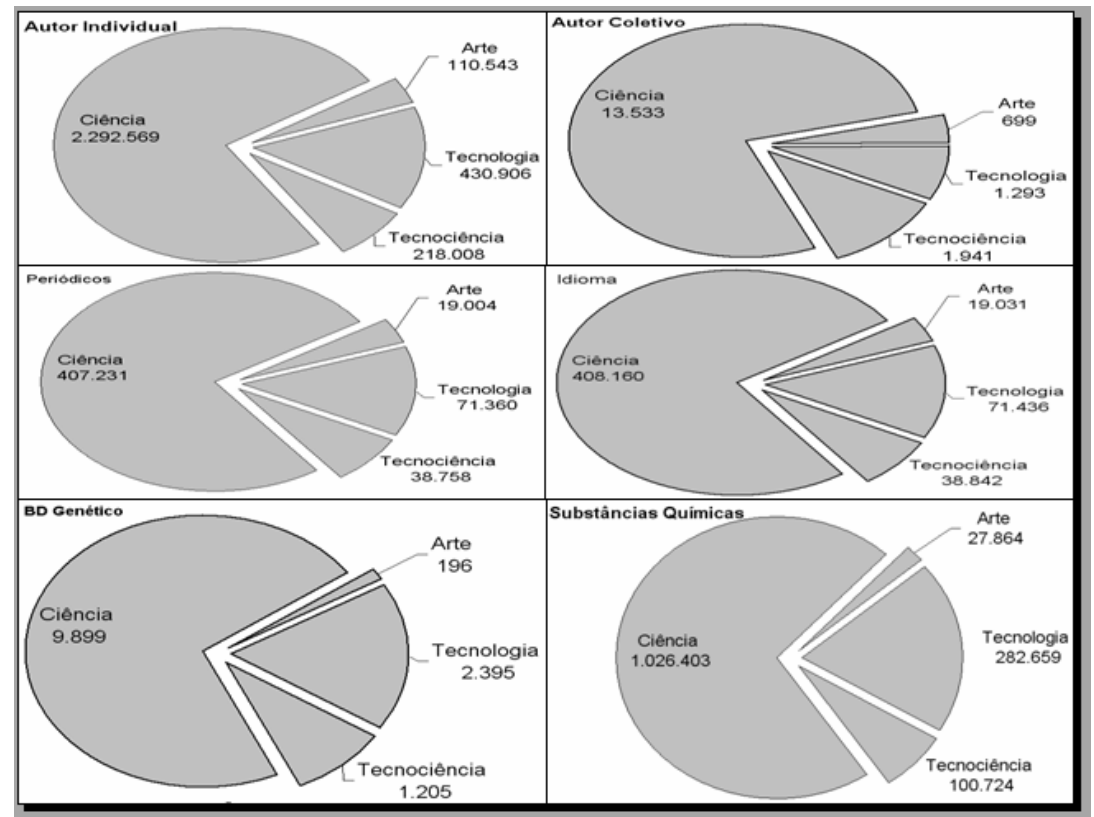

Figura 9 - Gráficos que relacionam o corpus ao tesauro EpistemIS quanto aos aspectos arte, ciência, tecnologia e tecnociência (1).

Também são apresentados gráficos evolutivos de artigos publicados por ano, país e tipo de publicação, e um gráfico que relaciona o ano e o país (Figura 10). Observa-se que o período de publicação dos artigos do corpus vai de 1997 a 2006 e a evolução anual das publicações é contínua, exceto em 2006, possivelmente porque o corpus foi coletado no início de 2007, quando provavelmente boa parte dos artigos de 2006 ainda não haviam sido inseridos na base PubMed. O idioma que mais contém publicações da IS é o inglês, em todos os aspectos, sucedido pelo espanhol e português. Os EUA publicam 10 vezes mais que todos os outros países do ranking separadamente. 
Uso da ferramenta PreText para mineração de textos extraídos do NCBI para estudo epistemológico da Informática em Saúde

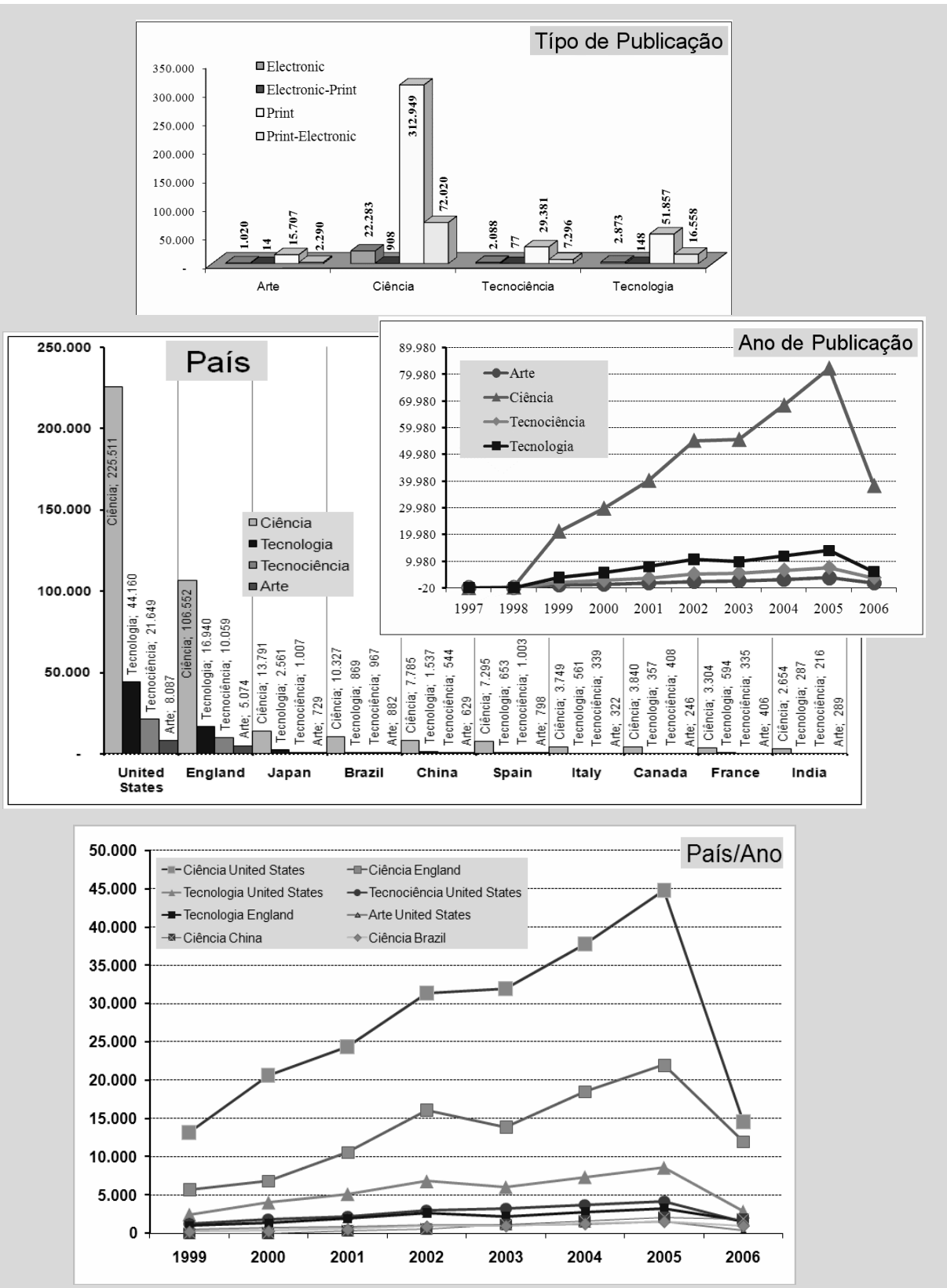

Figura 10 - Gráficos que relacionam o corpus ao tesauro EpistemIS quanto aos aspectos arte, ciência, tecnologia e tecnociência (2). 
Uso da ferramenta PreText para mineração de textos extraídos do NCBI para estudo epistemológico da Informática em Saúde

Um ranking de autores individuais (Tabela 1) que mais publicam em IS mostrou que a maioria dos autores é membro do Channing Laboratory, divisão de pesquisa multidisciplinar do Brigham and Women's Hospital e da Harvard Medical School, do EMGO Institute, VU University Medical Center Amsterdam e do Department of Ophthalmology and Visual Sciences da University of Wisconsin-Madison, USA.

Tabela 1 - Ranking de autores individuais que mais publicam em IS.

\begin{tabular}{|l|l|r|}
\hline \multicolumn{1}{|c|}{ MAPH } & \multicolumn{1}{|c|}{ Autor } & T. Registros \\
\hline Ciência & Willett, WCWalter C & $\mathbf{4 9 8}$ \\
\hline Ciência & Colditz, GAGraham A & $\mathbf{2 1 5}$ \\
\hline Ciência & Stampfer, MJMeir J & 194 \\
\hline Ciência & Mans on, JEJoAnn E & 193 \\
\hline Ciência & Wang, YY & 173 \\
\hline Ciência & Klein, RRonald & 163 \\
\hline Ciência & Hu, FBFrank B & 157 \\
\hline Ciência & Rimm, EBEric B & 152 \\
\hline Ciência & Wang, JJ & 152 \\
\hline Ciência & Hankinson, SESusan E & 148 \\
\hline
\end{tabular}

O ranking de autores coletivos (Tabela 2) mostrou que há muitos projetos integrados entre institutos de ensino e pesquisa e instituições em saúde, com ênfase em Saúde Pública, no cuidado em saúde e no controle e prevenção de doenças, ressaltando o aspecto tecnocientífico interdisciplinar da IS. Há ainda muitos grupos de pesquisa/trabalho, respectivamente, das áreas de Controle e Prevenção de Doenças (1.519), Ciências da Saúde e Farmacêuticas (241), Oncologia (107), Cardiologia (103), Saúde Materno-Infantil (100) e Cuidado em Saúde (93). Há uma tendência de autores coletivos atuarem na IS Pública.

Tabela 2 - Ranking de autores coletivos que mais publicam em IS.

\begin{tabular}{|c|c|c|c|}
\hline MAPH & Autor Coletivo & Área Atuação & T. Registros \\
\hline Tecnociência & Centers for Disease Control and Prevention (CDC) & Controle e Prevenção de Doenças & 125 \\
\hline Ciência & U.S. Preventive Services Task Force & Cuidado em Saúde & 93 \\
\hline Ciência & JACC Study Group (Japan Collaborative Cohort) & Oncologia & 74 \\
\hline Ciência & Framingham Heart Study & Cardiologia & 68 \\
\hline Ciência & Hedges Team & [diversos] & 63 \\
\hline Arte & Centers for Disease Control and Prevention (CDC) & Controle e Prevenção de Doenças & 46 \\
\hline Ciência & $\mathrm{CDC}$ & Controle e Prevenção de Doenças & 44 \\
\hline Ciência & EFCOSUM Group & Nutrição \& Saúde Pública & 35 \\
\hline Ciência & American Heart Association & Cardiologia & 35 \\
\hline Ciência & Atherosclerosis Risk in Communities study & Controle e Prevenção de Doenças & 32 \\
\hline Ciência & American College of Physicians & Medicina & 30 \\
\hline
\end{tabular}

O periódico que mais publica na área (Tabela 3) é o The Journal of Biological Chemistry (10.399), especializado em Bioquímica. Outras áreas representativas no ranking são: Microbiologia (18.294), Controle e Prevenção de Doenças (12.904), a própria Informática em Saúde (9.532), Neurociências (8.376), Radiologia (5.944) e especialidades 
Uso da ferramenta PreText para mineração de textos extraídos do NCBI para estudo epistemológico da Informática em Saúde

médicas, tais como Oncologia (9.384), Cardiologia (7.964), Pediatria (6.611), Endocrinologia (4.729). Na maioria dos periódicos prevalece o aspecto científico, com tendência para a Bioinformática. Os periódicos especializados em IS mais representativos são o Bioinformatics (Oxford, England) e o BMC Bioinformatics.

Tabela 3 - Ranking de periódicos que mais publicam em IS.

\begin{tabular}{|l|l|r|}
\hline \multicolumn{1}{|c|}{ MAPH } & \multicolumn{1}{|c|}{ Periodico } & T.Registros \\
\hline Ciência & The Journal of biological chemistry & 10.399 \\
\hline Ciência & Proceedings of the National Academy of Sciences of the United States of America & 7.129 \\
\hline Ciência & Pediatrics & 6.611 \\
\hline Ciência & Radiology & 5.944 \\
\hline Ciência & Bioinformatics (Oxford, England) & 5.893 \\
\hline Ciência & Journal of clinical microbiology & 5.598 \\
\hline Ciência & Chest & 5.368 \\
\hline Ciência & Clinical cancer research : official journal American Association Cancer Research & 5.222 \\
\hline Ciência & Nucleic acids research & 5.009 \\
\hline Ciência & Circulation & 4.934 \\
\hline Ciência & The Journal of clinical endocrinology and metabolism & 4.729 \\
\hline Ciência & The Journal of neuroscience : the official journal of the Society for Neuroscience & 4.384 \\
\hline Ciência & JAMA : the journal of the American Medical Association & 4.271 \\
\hline Ciência & Cancer research & 4.162 \\
\hline Tecnologia & The Journal of biological chemistry & 4.043 \\
\hline Ciência & Stroke; a journal of cerebral circulation & 3.992 \\
\hline Ciência & Applied and environmental microbiology & 3.644 \\
\hline Ciência & BMC bioinformatics & 3.639 \\
\hline Ciência & Blood & 3.580 \\
\hline Ciência & Diabetes care & 3.520 \\
\hline & & \\
\end{tabular}

\section{Discussão}

Pensava-se inicialmente que a IS fosse uma ciência aplicada interdisciplinar, embasada no método científico, que utiliza um corpo de conhecimento de outras disciplinas, tais como as Ciências da Saúde e a Computação. Após este trabalho, entende-se que a IS é uma ciência aplicada interdisciplinar, o que preferiu-se denominar tecnociência, a qual se ocupa da solução de problemas de um amplo leque de domínios e fatos das Ciências da Saúde e da Vida e da prática do Cuidado em Saúde, por meio da pesquisa científica interdisciplinar e do desenvolvimento de tecnologias próprias para uso na sociedade.

A ciência contida na Informática em Saúde é proveniente de sua base interdisciplinar e o seu corpo de conhecimento é composto por objetos e fatos de domínio tecnológico. A sua problemática concentra-se na resolução de problemas das Ciências da Saúde e da Vida, portanto, tecnocientífica.

Seu aspecto de ciência/tecnologia aplicada pauta-se em um conjunto de padrões, modelos, regras, normas e convenções que norteiam sua pesquisa e desenvolvimento. Há esforço da área em consolidar-se como ciência independente, mas é fortemente composta por conceitos interdisciplinares provenientes de outras ciências. O conjunto dos conceitos científicos da área que têm função na formação de suas teorias é pequeno, sendo a maioria de origem interdisciplinar. Seu aspecto artístico é mínimo e também interdisciplinar.

A IS apresenta desenvolvimento de seu próprio corpo de conhecimento, com ênfase tecnocientífica, tendo quantidade significativa de objetos e fatos de domínio que não lhe são próprios, mas que são foco da sua problemática. A área se ocupa em resolver problemas do 
Uso da ferramenta PreText para mineração de textos extraídos do NCBI para estudo epistemológico da Informática em Saúde

seu domínio, o que faz por meio da pesquisa científica e do desenvolvimento de tecnologias, buscando analisar a efetividade de suas soluções, usando métodos e técnicas de avaliação de áreas interdisciplinares.

Os principais objetos do domínio da Informática em Saúde são os dados, a informação e o conhecimento em Ciências da Saúde e da Vida. A problemática concentra-se no desenvolvimento de tecnologias e sistemas que contribuam com a gestão, aquisição, armazenamento, organização, recuperação e distribuição de dados, informação e conhecimento em saúde em qualquer suporte para o apoio à tomada de decisão na administração, educação e cuidado em saúde.

A nomenclatura que melhor se adequa para a área, embora não utilizada, é o termo Informática em BioSaúde, que dá conta da aplicação da Informática às Ciência da Vida ou Biológicas e às Ciências da Saúde.

O fato de a Informática em Saúde basear-se em ciências interdisciplinares não nos parece nenhum demérito, pois, na tentativa de construir uma ciência própria, recriando métodos que já existem sob algum neologismo para parecer genuína. Enquanto os elementos científicos de outras ciências são suficientes para que a IS cumpra seu papel de desenvolver tecnologias para o auxílio à saúde. Com esta preocupação a menos, a IS ganha agilidade no cumprimento de sua função tecnocientífica, beneficiando as Ciências da Saúde e a sociedade.

$\mathrm{O}$ fato de a IS ser uma tecnociência devido ao seu alto desenvolvimento tecnológico fundamentado em ciências interdisciplinares também não a torna menos benéfica ou essencial. Ao contrário, amplia seu valor para a sociedade. Além disso, uma tecnologia está sempre embasada por uma ciência, e tanto melhor se os fundamentos científicos da tecnologia presente na IS sejam provenientes de outras ciências já consolidadas. Afinal, a IS como área do conhecimento está apenas nascendo, então a sua interdisciplinaridade científica é o que dá sustentação ao seu desenvolvimento tecnológico, que herda das ciências de origem o rigor e a disciplina do método.

\section{Conclusão}

Com base nos resultados, concluímos que a IS é uma tecnociência interdisciplinar que se ocupa da solução de problemas de um amplo leque de domínios e fatos das Ciências da Vida e da Saúde e da prática do Cuidado em Saúde, por meio da pesquisa científica proveniente de outras áreas do conhecimento e do desenvolvimento de suas próprias tecnologias para uso na sociedade. 


\section{Referências}

1. MCCARTHY, J. What is artificial intelligence?. [s.n.]: Stanford University.Computer Science Department, nov./2004. Disponível em: <http://wwwformal.stanford.edu/jmc/whatisai/ whatisai.html>. Acesso em: 03 de março de 2007.

2. STERNBERG, RJ. The theory of successful intelligence. In: Interamerican Journal of Psychology - 2005, Vol. 39, Num. 2 pp. 189-202

3. Saussure, F. Curso de lingüística geral. São Paulo : Cultrix, 1974.

4. ALLEN, J. Natural language understanding. 2.ed. Redwood City : Benjamin/Cummings, 1995.

5. VIEIRA, R. ; Lima, VLS. Linguística computacional: princípios e aplicações. Porto Alegre : UNISINOS, [s.d]. Disponível em: $<$ http://www.inf.unisinos.br/ renata/laboratorio/ publicacoes/jaia12-vf.pdf $>$. Acesso em: 03 de março de 2007.

6. Ebecken, NF, Lopes, MCS, Costa, MCA. Mineração de textos. In: Sistemas inteligentes, Org.: Solange de Oliveira Rezende, Barueri, SP: Manole, 2003. p. 337-370.

7. Matsubara, ET. PreText: an environment for pre-processing text for text mining. Última atualização: 15/jun./2005. Disponível em: http://www.icmc.usp.br/ edsontm/pretext/ pretext.html. Acesso em: 13 de dezembro de 2006.

8. MARTINS, Cláudia A ; MONARD, M. C. ; MATSUBARA, E. T. . Uma metodologia para auxiliar na seleção de atributos relevantes usados por algoritmos de aprendizado no processo de mineração de textos. In: Conferencia Latinoamericana de Informatica, 2004, Arequipa. 30ma Conferencia Latinoamericana de Informática (CLEI2004). Peru : Sociedad Peruana de Computación, 2004. v. 1. p. 21-32.

9. MATSUBARA, ET. O algoritmo de aprendizado semi-supervisionado co-training e sua aplicação na rotulação de documentos. Dissertação (mestrado) orientada por Maria Carolina Monard no Instituto de Ciências Matemáticas e de Computação (ICMC) da Universidade de São Paulo. São Carlos, 2004. Disponível em: http://www.teses.usp.br/teses/disponiveis/55/55134/tde-19082004-092311. Acesso em: 6 de agosto de 2009.

10. COLEPÍCOLO, E. Epistemologia da Informática em Saúde: entre a teoria e a prática. Dissertação (mestrado) orientada por Ivan Torres Pisa no Departamento de Informática em Saúde da Universidade Federal de São Paulo. São Paulo, 2007.

11. BUNGE, M. Epistemologia: curso de atualização. 2.ed. Trad. Claudio Navarra. São Paulo: T.A.Queiroz, 1987. 246p. (Biblioteca de Ciências Naturais, v. 4)

12. PORTER, M. The Porter stemming algorithm. Última atualização: jan. 2006. Disponível em: <http://tartarus.org/ martin/PorterStemmer>. Acesso em: 21 out. 2007. 
Uso da ferramenta PreText para mineração de textos extraídos do NCBI para estudo epistemológico da Informática em Saúde

13. NIH. PubMed Central: a free archive of life sciences journal. Última atualização: 16 apr. 2007. Disponível em: <http://www.pubmedcentral.nih.gov>. Acesso em: 29 out. 2007.

14. NLM. MeSH: Medical Subject Headings. Washington: NLM, 2007. Disponível em: $<$ http://www.nlm.nih.gov/mesh/meshhome.html>. Acesso em: 3 out. 2007.

95. NLM/NIH/NBCI. Entrez Programming Utilities. Última atualização: 10 de agosto de 2006. Disponível em: http://eutils.ncbi.nlm.nih.gov/ entrez/query/static/eutils_help.html. Acesso em: 6 de agosto de 2009.

16. COLEPÍCOLO, E; HOLANDA, AJ; RUIZ, EES; WAINER, J; PISA, IT. MESH: de cabeçalho de assunto a tesauro. In: Congresso Brasileiro de Informática em Saúde, 10, 2006, Florianópolis/SC. Disponível em: <http://www.sbis.org.br/cbis/arquivos/994.pdf>. Acesso em: 6 de agosto de 2009.

17. VAN BEMMEL, J.H. (Ed.). Handbook of Medical Informatics. Rotterdam: Erasmus University, 1999. Última atualiação: 25 mar. 1999. Disponível em: <http://www. mieur.nl/mihandbook/r_3_3/ handbook/home.htm>. Acesso em: 29 out. 2007.

18. SHORTLIFFE, E.H.; CIMINO, J.J. Biomedical Informatics: computer Applications in Health Care and Biomedicine. 3.ed. New York: Springer, 2006.

19. BUNGE, M. La investigación científica. Barcelona, Ariel, 1969.

20. BUNGE, M. Ciência e desenvolvimento. Belo Horizonte/São Paulo: Itatiaia/EDUSP, 1980. $135 \mathrm{p}$. 